\title{
Lexis
}

Journal in English Lexicology

$5 \mid 2010$

Lexicology \& Stylistics

\section{Exploitation stylistique du processus de composition nominale en anglais contemporain}

\section{Elise Mignot}

\section{(2) OpenEdition}

\section{Journals}

Édition électronique

URL : http://journals.openedition.org/lexis/472

DOI : $10.4000 /$ lexis.472

ISSN : 1951-6215

Éditeur

Université Jean Moulin - Lyon 3

Référence électronique

Elise Mignot, «Exploitation stylistique du processus de composition nominale en anglais

contemporain », Lexis [En ligne], 5 | 2010, mis en ligne le 15 octobre 2010, consulté le 23 avril 2019. URL : http://journals.openedition.org/lexis/472 ; DOI : 10.4000/lexis.472

\section{(c) (i) (9)}

Lexis is licensed under a Creative Commons Attribution-NonCommercial-NoDerivatives 4.0 International License. 


\title{
Exploitation stylistique du processus de composition nominale en anglais contemporain
}

Elise Mignot ${ }^{1}$

\begin{abstract}
We focus on a few English compound nouns stylistically marked in that they are non conventional denominations. These compounds are also characterized by their shortness, in as much they synthetize an underlying discourse. We compare compound nouns to compound adjectives, and it comes out that compound adjectives are much less characterized by shortness, even if they can also be seen as the synthetic form of an underlying analytic structure. If it can be assumed that these remarks pertaining to the domain of word-formation give us some insight into nouns and adjectives in general, shortness then appears to be a characteristic of nouns rather than adjectives. Our initial stylistic approach leads us to a reflexion on grammatical categories.
\end{abstract}

Keywords: stylistics - compound nouns - parts of speech

\section{Résumé}

Nous nous penchons sur quelques noms composés anglais qui sont stylistiquement marqués dans la mesure où ils disent un référent de façon non conventionnelle. Ces composés sont par ailleurs caractérisés par la brièveté, car ils condensent un discours sous-jacent. Nous comparons les noms composés aux adjectifs composés, et il ressort que ces derniers, même s'ils peuvent également être vus comme synthétisant une structure analytique sous-jacente, ne présentent pas le même caractère de brièveté ou du moins, pas au même degré. Si l'on admet que ces remarques appartenant au domaine de la formation des mots nous renseignent, plus généralement, sur les parties du discours nom et adjectif, la brièveté apparaît alors comme caractérisant la catégorie nominale plutôt qu'adjectivale. Notre comparaison sytlistique nous mène ainsi à une réflexion sur les parties du discours.

Mots-clés : stylistique - noms composés - parties du discours

\footnotetext{
${ }^{1}$ Université Sorbonne - Paris 4 : Elise.Mignot@paris-sorbonne.fr
} 


\section{Introduction}

Nous nous intéressons à l'exploitation stylistique du processus de composition nominale (de type nom + nom, avec un accent intial) en anglais contemporain. Les exemples et statistiques proviennent d'un corpus de 1097 noms composés, tous relevés en contexte et provenant de sources variées (romans, pièces de théâtre, scénarios de film, conversations, émissions de télévision $)^{2}$. Ce processus de formation des noms est très productif : dans notre corpus plus de la moitié des items (53\%) n'apparaissent pas dans le dictionnaire. Ce sont tout particulièrement ces derniers (les noms composés non lexicalisés) qui sont porteurs d'effets de style. Dans notre corpus la proportion de noms composés non lexicalisés qui font l'objet d'une exploitation stylistique est de 5 à $10 \%$, selon que l'on retienne les items présentant les trois critères que nous envisagerons (dénomination non standard, effet de connivence, brièveté), ou ceux qui présentent seulement l'un d'eux. Précisons tout d'abord en quoi consistent ces effets de style.

\section{Dénominations non standard et connivence}

\subsection{Le style vu comme un écart par rapport à une norme}

Une dénomination non standard s'oppose à une dénomination standard, communément acceptée ; par cet écart même, elle est stylistiquement marquée. Nous retenons ici la définition du style, comme écart par rapport à une norme, et non pas nécessairement effet de littérarité, telle que la rappelle Molinié [1986 : 11] :

[...] c'est en gros la tradition de Bally. On part du principe que, dans la pratique du langage, on peut isoler des segments de discours, identifier des faits langagiers, et traduire de diverses façons des contenus sémantiques identiques. Par rapport à une espèce de degré zéro d'expression [...] on délimite un écart dans le discours occurrent. ${ }^{3}$

Le même auteur [1986:18] précise que lorsqu'on se situe à l'échelle du mot, comme c'est le cas ici, un des paramètres à prendre en compte lors de l'analyse stylistique est donc la sensibilité au caractère banal, commun du vocabulaire (entendu comme somme des mots d'un ensemble), dû à la simple reprise de l'arsenal utilisable simultanément par la totalité des groupes sociaux d'une époque donnée, ou, au contraire, à des marques de différenciation, de résonnance, de renouvellement [...].

Les noms composés que nous envisageons s'écartent de la norme dans la mesure où ils n'appartiennent pas au lexique commun à l'ensemble des locuteurs d'une communauté linguistique. Dans notre corpus ce décalage est associé à l'humour. A titre de comparaison, nous avons effectué des relevés dans deux sources, l'une au registre clairement humoristique, le film Annie Hall de Woody Allen, l'autre ne présentant pas cette caractéristique, le roman Wuthering Heights de Emily Brontë ${ }^{4}$. La proportion de noms composés par rapport à

\footnotetext{
${ }^{2}$ Le corpus est issu de notre thèse de doctorat [Mignot 2001], mais les faits et idées présentés ici (l'exploitation stylistique des noms composés) ne figurent pas dans la thèse.

${ }^{3}$ Nous soulignons.

${ }^{4}$ Le choix de ces œuvres répond à des considérations pratiques. Annie Hall est l'une des œuvres dans lesquelles nous avions déjà fait un relevé exhaustif des noms composés. Wuthering Heights a été choisi de façon plus arbitraire, simplement car ce roman, parmi beaucoup d'autres certes, n'est pas écrit dans un style humoristique où l'on recherche les dénominations non standard.
} 
l'ensemble des noms est sensiblement la même dans les deux cas (4 et 3\% respectivement), mais dans Wuthering Heights, seulement 11\% des noms composés sont des formations de discours (n'apparaissent pas dans le dictionnaire), tandis que cette proportion atteint $50 \%$ dans Annie Hall. Les quelques formations de discours qui apparaissent dans Wuthering Heights (coffin lid, moor sheep par exemple) n'apparaissent en outre pas du tout comme des dénominations décalées (c'est-à-dire s'écartant de la norme), tandis que cela est fréquemment le cas dans Annie Hall ${ }^{5}$. On observe donc une corrélation entre composition nominale (non lexicalisée) et texte humoristique.

Cet effet de style (la dénomination décalée associée à l'humour) n'est certainement pas le seul que l'on puisse attribuer aux noms composés (il existe aussi, par exemple, un emploi poétique) mais c'est celui sur lequel nous nous concentrerons ici.

Nous présenterons donc ces dénominations décalées en précisant d'emblée le corollaire de ce phénomène : si les dénominations que nous envisageons n'appartiennent pas au lexique commun, elles ne valent que pour quelques locuteurs, et non pas pour tous, ce qui tend à produire entre eux un effet de connivence, que nous nous attacherons également à décrire.

\subsection{Une interprétation personnelle du référent}

Dans les deux exemples suivants, tirés de la bande dessinée Calvin and Hobbes, un petit garçon fait part à son tigre en peluche (un ami imaginaire) de ses soucis à propos des cadeaux de Noël.

This Santa Claus stuff ${ }^{6}$ bothers me... especially the judge and jury bit. $^{7}$ [Waterson $1990: 105]$

This whole Santa Claus thing just doesn't make sense. [Waterson 1990 : 106]

L'enfant est préoccupé par un type de discours sur le père Noël : les enfants pas sages ne reçoivent pas de cadeaux. La dénomination judge and jury bit n'est pas standard dans la mesure où il n'est pas habituel de parler du Père Noël comme d'un juge qui enverrait les enfants au tribunal, et l'on comprend qu'il s'agit là d'une interprétation de la situation par l'enfant. C'est ce décalage qui produit un effet de drôlerie, décalage d'autant plus marqué que pour subjective qu'elle soit, cette dénomination se donne comme tout à fait normale (nous reviendrons sur ce point). La situation (un petit garçon parle à son tigre en peluche, auquel il prête vie et parole) ne fait que mettre en valeur cet aspect décalé : le petit garçon est dans son monde. Le caractère non standard de la dénomination apparaît également dans l'exemple suivant, tiré de la même bande dessinée, avec encore une fois un effet d'humour. Il s'agit d'un échange entre un petit garçon et son père.

- Bad news on your polls, Dad. You dropped another 5 points. It seems that although your recognition factor is high, the scandals of your administration continue to haunt you.

- Scandals? What scandals?!

\footnotetext{
${ }^{5}$ Il convient toutefois de traiter ces statistiques avec prudence. Si, dans le cas de Annie Hall, le décompte a été réalisé sur l'ensemble de l'œuvre, sur Wuthering Heights, le relevé est moins exhaustif puisqu'il n'a été effectué qu'à titre de comparaison et ne porte que sur deux chapitres du roman.

${ }^{6}$ On voit que dans notre relevé des composés de type nom + nom nous avons inclus des items dont les constituants sont eux-mêmes complexes. Dans Santa Claus stuff, le N1 est le nom propre (Santa Claus) et le N2 stuff. Notre seul critère était le statut nominal de chacun des deux constituants, et le fait que l'ensemble fonctionne comme un seul nom (c'est-à dire puisse fonctionner comme tête d'un syntagme nominal, et être précédé d'un déterminant).

${ }^{7}$ Nous indiquons en gras les noms composés qui font l'objet de notre propos.
} 
- Bedtimegate and homeworkgate come readily to mind. [Waterson 1990 : 154$155]$

Le petit garçon s'adresse à son père comme si ce dernier était candidat à une élection (de type élection présidentielle, mais ici pour devenir le père du petit garçon). Nous traitons bedtimegate et howeworkgate comme des composés ayant pour tête gate, formés sur le modèle de Watergate, mais où gate a acquis la signification de scandal. L'Oxford English Dictionary décrit ainsi cet élément :

Forming nouns denoting an actual or alleged scandal (and usu. an attempted cover-up) comparable in some way to the Watergate scandal of 1972, esp. with the name of the place associated w. the scandal, as Dallasgate, Irangate.

Même si la réalité nommée existe (il y a bien conflit entre le petit garçon et son père au sujet de l'heure du coucher et des devoirs), la dénomination est décalée car elle donne l'impression de désigner un problème beaucoup plus important et largement connu que celui qu'évoque le petit garçon.

\subsection{Cas où le référent n'existe pas}

Parfois la dénomination est non standard, non pas parce qu'il en existe une autre, plus standard, mais parce que le référent n'existe pas. Elle est alors également empreinte d'humour, comme en témoignent les deux exemples suivants, tirés respectivement des films Manhattan et Annie Hall :

IKE (Interrupting). This is terrible. I, uh, you know, I, listen, you shouldn't ask me for advice. I... (Yale sighs). When it comes to relationships with women, I'm the winner of the August Strinberg Award. [Allen 1983 : 186]

ALVY [...] And there's the winner of the Truman Capote look-alike contest. ${ }^{8}$ [Allen $1983: 46$ ]

Le locuteur sait bien qu'il n'existe pas de telles réalités (le concours August Strindberg permettant de distinguer l'homme ayant le plus de difficultés à mener une vie de couple, le concours des sosies de Truman Capote), mais fait comme si elles existaient, et même comme si elles étaient connues de son interlocuteur. Là encore, l'effet humoristique, pour l'interlocuteur mais aussi pour le spectateur du film, provient du fait que la dénomination se donne comme non problématique, alors qu'en réalité elle n'est pas conventionnelle.

\subsection{L'effet de connivence}

Dans l'exemple déjà cité de the Truman Capote look-alike contest, on l'a vu, la réalité désignée n'existe pas. Elle n'a donc a priori pas de nom. Le fait de la nommer (par un nom ad $h o c$ ), en plus de présupposer son existence, crée une connivence entre l'énonciateur et l'interlocuteur car le nom n'est pas connu de l'ensemble de la communauté linguistique. Il ne fonctionne que pour les deux interlocuteurs et contribue à créer entre eux une complicité. Dans cet exemple, comme dans celui de the August Strindberg Award, l'effet de connivence est renforcé par le fait qu'il faut être assez cultivé pour comprendre ce que serait la réalité

\footnotetext{
${ }^{8}$ La forme Truman Capote look-alike contest présente un cas de récursivité. Le premier élément (Truman Capote look-alike) est lui-même un composé. Encore une fois, notre seul critère était le statut nominal de chacun des deux constituants, et le fait que l'ensemble fonctionne comme un seul nom.
} 
désignée si elle existait, en l'occurrence connaître August Strindberg, savoir que ses pièces de théâtre aussi bien que sa vie personnelle sont caractérisées par des relations de couples difficiles. On a là un effet de snobisme, qui n'est d'ailleurs pas réservé aux cas où la réalité désignée n'existe pas. Il apparaît également dans :

IKE. [...] It's an interesting group of people your friends are.

MARY Oh, I know

IKE It's like a cast of a Fellini movie. [Allen $1983: 206$ ]

IKE This - this is shaping up like a Noel Coward play, you know. Somebody should go out and make some martinis. [Allen 1983 : 262]

Il faut connaître Fellini et Noel Coward pour pouvoir interpréter ces noms composés : les dénominations permettent, indirectement, de souligner la connivence d'une petite communauté d'intellectuels, new-yorkais en l'occurrence. Précisons que dans ces deux derniers exemples l'effet de connivence n'est pas lié au fait que la dénomination est surprenante. On peut néanmoins la qualifier de non standard dans la mesure où elle n'est pas accessible à l'ensemble de la communauté linguistique.

\section{La brièveté}

Outre leur aspect décalé, une caractéristique de ces dénominations est la brièveté. Les noms composés sont courts, non pas par rapport à d'autres types de noms bien sûr, mais par rapport à un discours sous-jacent, explicite ou non, qu'ils condensent. Rappelons que la prise en compte de la volumétrie est l'un des outils de l'analyse stylistique.

\subsection{Volume et stylistique}

Dans son premier chapitre, consacré au mot, Molinié [1986 : 16] mentionne le volume comme tout premier critère d'analyse. L'étude volumétrique n'a d'intérêt que si elle est différentielle. Bien sûr il $\mathrm{y}$ a des mots «longs» et des mots «courts» : anticonstitutionnellement - oui ; et la plupart des lexies n'appellent aucun commentaire de ce point de vue. Mais il y a surtout les mots qui changent de volume selon la prononciation : on peut prononcer oui ou non aussi longtemps que des termes de plusieurs syllabes; on peut raccourcir, par abréviation ou par accélération de débit, les mots volumineux. Enfin, et c'est le plus important, l'impression volumétrique reçue peut varier par opposition contextuelle. Le sortez de Roxane qui interrompt l'assez long discours embarrassé et dilatoire de Bajazet, en le vouant ainsi, en principe, aux exécuteurs, doit en réalité occuper un espace sonore aussi considérable, pour le public, que l'ensemble des dizaines d'alexandrins débités par Bajazet: on pourrait même imaginer une mise en scène qui transformerait peu à peu les tirades du héros en pur bruitage, lequel serait brutalement suivi de la prononciation distinctive de deux syllabes, amplifiées par le synthétiseur.

Ces quelques lignes nous permettent d'une part de rappeler la pertinence du critère du volume en analyse stylistique, d'autre part de préciser notre façon d'utiliser ce paramètre. L'idée maîtresse est celle du caractère relatif de l'analyse volumétrique, et il nous faut donc préciser à quoi nous comparons les noms composés. Il n'est pas question ici de réalisation phonique, ni de comparaison avec d'autres types de noms. Le volume des noms composés est comparé à celui du discours qui sous-tend ces composés. 


\title{
2.2. Un discours sous-jacent
}

Le nom composé paraît bref par rapport à un discours qu'il condense. L'exemple suivant est tiré de The Loneliness of a Long Distance Runner. Il permet de constater qu'une dénomination (Telly Boys) qui ne vaut que pour quelques-uns (les jeunes gens et la mère du narrateur), une dénomination non standard, donc, consiste en la réduction d'un discours antérieur.

\begin{abstract}
And it was when these cops were chasing the crooks that we played some good tricks with the telly, because when one of them opened his big gob to spout about getting their man I'd turn the sound down and see his mouth move like a goldfish or mackerel or a minnow mimicking what they were supposed to be acting - it was so funny the whole family nearly went into fits on the brand-new carpet that hadn't yet found its way to the bedroom. It was the best of all though when we did it to some Tory telling us about how good his government was going to be if we kept on voting for them - their slack chops rolling, opening and bumbling, hands lifting to twitch moustaches and touching their buttonholes to make sure the flower hadn't wilted, so that you could see they didn't mean a word they said, especially with not a murmur coming out because we'd cut off the sound. When the governor of the Borstal first talked to me I was reminded of those times so much that I nearly killed myself trying not to laugh. Yes, we played so many good stunts on the box of tricks that mam used to call us the Telly Boys, we got so clever at it. [Sillitoe $1959: 22-23$ ]
\end{abstract}

Si ce processus de condensation est parfois exploité à des fins stylistiques (la brièveté est alors mise en valeur et signifiante - nous revenons sur ce point en 2.4. - il concerne en fait tous les noms composés. Ainsi, O'Neill book dans I've got to get this O'Neill book finished [Allen 1983 : 187] est préconstruit par I'm writing a book about O'Neill. Dans ce dernier exemple le déterminant défini this est d'ailleurs un indice du discours ancien, synthétisé dans le composé. De même dans l'exemple suivant, l'adjectif old ne dit pas l'âge du référent mais le fait qu'il a déjà été mentionné, et que les deux éléments du composé ont déjà été réunis dans un discours préalable.

In the morning old Hitler-face questioned me again. [Sillitoe $1959: 35$ ]

Hitler-face réfère en effet à un policier, qui, dans le co-texte avant, a été explicitement comparé à Hitler :

He was like Hitler in the face, right down to the paint-brush tash, except that being six-foot tall made him seem worse. [Sillitoe 1959 : 31]

Notons en outre que dans cet exemple le premier élément est un nom propre ; cela est fréquent dans notre corpus et témoigne également du fait que ces noms composés condensent un discours.

\subsection{Les noms propres}

A examiner le corpus, on est frappé de la fréquence des cas où le premier élément est un nom propre. En voici quelques exemples, pour certains déjà cités : 
And there's the winner of the Truman Capote look-alike contest. [Allen 1983 : 46]

I was at an Alice Cooper thing where six people were rushed to hospital with bad vibes [Allen $1983: 66$ ]

When it comes to relationships with women, I'm the winner of the August

Strinberg Award. [Allen 1983 : 186]

This - this is shaping up like a Noel Coward play, you know. Somebody should go out and make some martinis. [Allen 1983 : 262]

If you don't get a good night kiss, you get Kafka dreams. [Waterson 1990 : 87]

Dans tous les cas le nom propre est un nom de personne célèbre, et sort de son rôle de " désignateur rigide » [Kripke 1980] pour condenser un discours : la raison pour laquelle la personne est célèbre, ce que l'on sait à son sujet. On se doute que le concours des sosies de Truman Capote ne va pas sélectionner les gens les plus beaux. A Alice Cooper sont associées un certain nombre de caractéristiques (un chanteur excentrique, un style de musique), de même qu'à August Strindberg (des relations compliquées avec les femmes, que ce soit dans sa vie ou dans son œuvre). De façon similaire, Noel Coward est associé à la mondanité, la superficialité, le dandyisme. Dans Kafka dreams, on comprend que les rêves ne sont pas agréables, qu'il s'agit plutôt de cauchemards. On associe à Kafka les idées d'absurdité et de peur. Rappelons également l'exemple de Hitler-face utilisé pour désigner un policier, où le premier nom ne condense évidemment pas un discours positif et traduit l'hostilité du narrateur envers ce personnage. Dans l'exemple suivant, Bambi ne réfère pas à une personne mais à un animal célèbre, et la logique est la même :

I don't think giving her Bambi eyes is going to get you a flame thrower.

[Waterson $1990: 31$ ]

Un petit garçon, Calvin, s'entraîne devant le miroir à faire les yeux doux à sa mère pour qu'elle lui achète le jouet de ses rêves (un lance-flamme). Son tigre en peluche (et ami imaginaire) exprime ses doutes quant au succès de son stratagème. Dans Bambi eyes est condensé un discours sur Bambi, c'est-à-dire la représentation que nous avons de cet animal (un faon aux yeux doux qui ne ferait de mal à personne).

\subsection{Brièveté et caractère non problématique de la dénomination}

Rappelons que même si les dénominations que nous avons envisagées sont marquées stylistiquement car non conventionnelles, elles se présentent néanmoins comme non problématiques. Nous mettrons ici en relation la brièveté des noms composés (par rapport au discours qui les sous-tend) et le caractère non problématique de la dénomination.

Ainsi, les composés sont synthétiques parce que le rapport qui unit les deux termes du composé est acquis, aussi bien par l'énonciateur que par le co-énonciateur. Hitler-face est possible parce que le narrateur a exposé ses raisons de comparer le policier à Hitler. De même, lorsque l'ami imaginaire du petit garçon Calvin évoque the salamander incident, les deux protagonistes savent de quoi il retourne (tandis que le lecteur, lui, ne le sait pas, et est amené à imaginer les bêtises les plus farfelues). Le composé Telly Boys est lui aussi compréhensible pour les garçons et leur mère parce que tous connaissent les farces des jeunes gens devant la télévision; il est en outre interprétable par le lecteur car le narrateur a préalablement exposé l'explication de cette dénomination.

Les noms composés peuvent donc apparaître si la dénomination est partagée, fût-ce par un petit nombre, et que donc elle ne pose pas problème. Dans les quelques lignes qui suivent, 
Levi [1978 : 61] délaisse brièvement son approche générativiste pour évoquer la fonction de dénomination des noms composés.

For example, although the transitory shape of a cloud may be of little interest to most speakers of English, it could have "classificatory relevance" to members of a sect which interprets animal-shaped clouds as omens of great significance; Zimmer thus claims $(1971: \mathrm{C} 14)$ that the sight of a cloud shaped like a kangaroo might lead speakers of the former group to utter (3.9a), but that only the speakers of the latter group would be led to coin a new compound by uttering (3.9b).

a. Hey, there's a cloud that looks like a kangaroo!

b. Hey, look at that kangaroo cloud. ${ }^{9}$

Nous relèverons que, pour imaginer un contexte où l'exemple kangaroo cloud puisse apparaître, son auteur pense à une " secte " (c'est-à-dire à une communauté). Ce composé, aussi improbable soit-il, est aisément interprétable si le principe de classification des nuages est partagé. Même si la dénomination n'est pas conventionnelle, au sens où elle n'est pas partagée par l'ensemble d'une communauté linguistique, elle est communautaire, c'est-à-dire partagée par quelques-uns. L'effet stylistique de décalage surgit lorsque le nom composé est entendu par un individu qui n'appartient pas à la communauté pour laquelle la dénomination fonctionne, et cet effet contribue à faire ressortir la brièveté du composé. Cette dernière traduit en effet le caractère non problématique de la dénomination, car on développe normalement ce qui ne va pas de soi : ce qui demande une explication, ou ce sur quoi on n'est pas d'accord. Lorsqu'est prononcé the Truman Capote look-alike contest, l'énonciateur fait comme si la dénomination ne posait aucun problème (alors que ni la réalité désignée ni la communauté pour qui la dénomination est interprétable n'existent), et c'est la brièveté même de la dénomination qui indique son caractère censément non problématique. De fait, même lorsqu'un composé est objectivement long, comme c'est le cas ici (long par rapport à un nom simple, ou même par rapport à d'autres noms composés, tels que milkman), il paraît d'autant plus bref que le référent n'existe pas. En effet un référent surprenant requiert a priori une présentation et une description. Downing [1977 : 822] rappelle la différence entre la fonction des noms composés et celle des phrases :

Most authors have, of course, recognized that, unlike full sentences, compounds serve to refer rather than to assert. That is, a speaker who utters the sentence John dropped a wine glass commits himself to the existence of an individual named John and a class of entities named wine glasses, and to the truth of the proposition $X$ dropped $\mathrm{Y}$ (where $X=J o h n$ and $Y=$ wine glass). He takes for granted the proposition $W$ is designed to hold $Z$, or $W$ is intended for $Z$, or some similar proposition (where $W=$ glass and $Z=$ wine), but he does not assert it.

Les noms composés sont brefs car ils ne servent pas à asserter mais à nommer; ils sont nécessairement communautaires, tandis que la phrase est individuelle.

Reste que tous les noms (et non pas seulement les noms composés) servent à nommer, et que, si la brièveté est le corrolaire de cette fonction de dénomination, on peut s'attendre à ce que les noms soient, de façon générale, plus courts que les représentants d'autres parties du discours, comme les adjectifs. S'il n'est peut-être pas très pertinent de comparer la longueur de tous les noms et de tous les adjectifs en comptant, par exemple, les phonèmes ou les syllabes, le domaine de la formation des mots apparaît comme un terrain privilégié pour tester cette hypothèse. Nous comparerons donc les noms composés aux adjectifs composés, du point

\footnotetext{
${ }^{9}$ Nous soulignons.
} 
de vue de leur longueur par rapport à leurs structures sous-jacentes respectives (dans la mesure où tous deux semblent issus de constructions plus développées). Il est possible que ces remarques, qui relèvent du domaine de la formation des mots, nous permettent d'affiner notre compréhension des catégories nominale et adjectivale, au delà des phénomènes de composition. Nous nous orientons ainsi vers une réflexion sur les parties du discours.

\section{Composition nominale et composition adjectivale}

D'un point de vue stylistique, la composition adjectivale ne semble pas posséder les mêmes caractéristiques que la composition nominale. De façon générale, les adjectifs composés ne semblent pas être particulièrement utilisés à des fins humoristiques. Pour vérifier cette hypothèse nous avons effectué un relevé des adjectifs composés dans les films Manhattan et Annie Hall, ainsi que dans quelques pages de la bande dessinée Calvin and Hobbes (75 items au total), œuvres dans lesquelles nous avions également relevé tous les noms composés (de type nom + nom, recevant un accent initial), et qui sont caractérisées par un registre humoristique. Nous reconnaissons le caractère hétérogène de notre liste d'adjectifs composés, mais, ces derniers n'étant pas très nombreux, nous avons souhaité les inclure tous afin de vérifier plus facilement d'une part le fait que les adjectifs composés ne présentent pas le même caractère non standard que les noms composés, d'autre part qu'ils ne sont pas associés à l'impression de brièveté ${ }^{10}$.

\subsection{Les adjectifs composés plus conventionnels que les noms composés}

A première vue les adjectifs composés ne sont pas très différents des noms composés : on observe la même proportion par rapport aux formes simples ( $4 \%$ dans les deux cas ${ }^{11}$ ), et une faible différence en ce qui concerne le taux d'inscription dans le dictionnaire (47\% pour les noms composés, $45 \%$ pour les adjectifs composés). Ces $45 \%$ s'élèvent cependant à $55 \%$ si l'on prend en compte les adjectifs composés qui n'apparaissent pas dans le dictionnaire comme tels mais qui sont formés à partir d'une base présente dans le dictionnaire (crewcutted à partir de crew cut, back-lit à partir de to back light par exemple). Ces chiffres indiquent que la composition adjectivale est un peu moins sujette à la création lexicale. Or on peut s'attendre à ce que la catégorie la moins sujette à la création lexicale (en l'occurrence la catégorie adjectivale) soit celle dans laquelle on retrouve le plus de signes conventionnels, c'est-à-dire de signes utilisables et interprétables par l'ensemble d'une communauté linguistique, et stylistiquement non marqués.

De fait les adjectifs composés semblent dire surtout des caractéristiques " objectives » (c'est-à-dire observables par tous) du référent. En témoignent les nombreux composés en -less très descriptifs : toothless, motionless, ainsi que les lexèmes de couleur (qui apparaissent pas exemple dans black-rimmed, pitch black, white-clothed), ou encore les numéraux (comme dans six-year old). La très grande majorité de ces adjectifs (96\%) sont d'ailleurs non scalaires : ils disent des propriétés censées être soit présentes, soit absentes, qui ne résultent pas d'une évaluation personnelle. Nous n'avons en fait qu'une forme qui soit scalaire : old fashioned (qui apparaît trois fois). Précisons d'ailleurs que cet adjectif est scalaire en théorie, mais que dans les contextes où nous l'avons relevé, il n'apparaît pas de forme de gradation (par exemple dans le syntagme nominal : an old-fashioned, cluttered, doctor's office). Dans

\footnotetext{
${ }^{10}$ Les statistiques concernant les adjectifs composés, ayant été conduite dans l'optique d'une comparaison avec les noms composés, ont été effectuées sur des corpus beaucoup plus restreints. Il convient donc de les traiter avec une certaine prudence.

${ }^{11}$ Le décompte a été effectué sur l'ensemble du scénario de Annie Hall.
} 
notre corpus, nous n'avons donc aucun adjectif composé associé à une forme de gradation. Le caractère non scalaire de ces adjectifs est d'autant plus marqué que dans certains cas leur deuxième élément, pris isolément, le serait. Ainsi ice cold n'est pas scalaire alors que cold l'est; de même pour street-smart par opposition à smart. On observe également dans cette perspective qu'un élément tel que well, qui serait évaluatif utilisé seul (dans un autre contexte) ne l'est pas dans well-worn.

Ces adjectifs, étant très descriptifs, ne proposent pas une interprétation individuelle du référent. Ils disent des propriétés visibles par tous, qu'il ne paraît pas surprenant d'attribuer au référent. Nous avons vu que les noms composés, au contraire, disent parfois des réalités surprenantes, voire inexistantes, contredisant d'ailleurs la "contrainte de l'existence » (requirement of existence) mentionnée par Bauer [1983: 85] :

As a general rule, a word will not be formed to denote an item/action/quality which does not exist.

La contrainte de l'existence semble en fait valoir plus pour les adjectifs que pour les noms. Il paraît plus facile d'inventer des réalités nominales (comme en témoigne Truman Capote lookalike contest) que des qualités, et on observe en effet que les adjectifs composés disent parfois des propriétés absentes (toothless), mais pas des propriétés inexistantes. On n'a donc pas l'équivalent dans le domaine adjectival de l'effet rencontré chez les noms composés, où l'on dit une réalité qui n'existe pas, tout en faisant comme si elle existait, et comme si sa dénomination ne posait aucun problème. Nous avons vu à propos des noms que le caractère non problématique de la dénomination était lié à la brièveté relative du nom composé. Voyons ce qu'il en est des adjectifs composés de ce point de vue.

\section{2. Comparaison entre noms composés et adjectifs composés du point de vue de la brièveté}

Les adjectifs composés ne présentent pas le même caractère de brièveté que les noms composés. Ils sont synthétiques, par rapport à une structure syntaxique plus développée, mais le phénomène de réduction paraît beaucoup moins poussé que pour les noms composés. D'un point de vue sémantique, les adjectifs composés ne paraissent pas chargés d'implicite comme le sont leurs contreparties nominales.

Ainsi, dans notre corpus, nous n'avons pas d'adjectif composé qui soit ambigu pour d'autres personnes que l'énonciateur et le co-énonciateur, comme l'est par exemple salamander incident. On peut en effet interpréter ce dernier de multiples façons : the salamander that you found in the garden and brought into the house, ou même, connaissant l'inventivite du petit garçon, the salamander that you put in your Mum's soup (le contexte nous indique qu'il s'agit d'une bêtise). De façon générale, les noms composés sont éminemment caractérisés par l'ambiguïté, non pas au sens où ils sont ambigus pour l'énonciateur et le co-énonciateur, mais au sens où ils le sont pour d'autres personnes. Ainsi le composé Telly Boys, évoqué plus haut, n'est interprétable que dans son contexte, où il réfère à des jeunes gens qui s'amusent à couper le son de la télévision et à imiter la gestuelle des présentateurs. Dans ce composé sont condensés plusieurs éléments lexicaux, comme mimick par exemple (verbe qui apparaît dans le co-texte avant). Dans un autre contexte, le même composé pourrait renvoyer à un tout autre type de personnes, par exemple les jeunes gens qui passent à la télévision, qui vendent des télévisions, qui en réparent... Et, hors contexte, ce composé serait difficilement interprétable. Les adjectifs composés quant à eux ne paraissent pas présenter les mêmes difficultés d'interprétation pour peu que l'on connaisse le sens de leurs composants. Dans un adjectif composé, en général, il ne semble pas «manquer» 
d'élément lexical. Ainsi, dans spotlighted, tout est dit avec les deux éléments lexicaux, spot et light. De même le terme high-pitched, pour être expliqué, ne requiert pas d'autre élément lexical. Contrairement à ce qu'on a observé pour les noms composés, aucun nom propre de personne célèbre n'entre d'ailleurs dans la formation des adjectifs composés. On ne peut donc évoquer un discours condensé dans les adjectifs composés, du moins par ce biais. Ces diverses remarques indiquent que les adjectifs composés, contrairement aux noms composés, ne condensent pas d'éléments lexicaux autres que ceux qui apparaissent dans leur forme, et c'est en ce sens que nous affirmons qu'ils sont, relativement parlant, moins brefs que les noms composés. Le seul contre-exemple éventuel que nous avons relevé est crew-cutted, qui permet de dire une coupe de cheveux, et dans lequel on peut penser que l'élément hair est implicite : les cheveux de la personne en question sont coupés comme ceux des marins d'un équipage (crew) de bateau. Notons toutefois que ce composé est répertorié dans le dictionnaire, et que cela atténue son caractère ambigu, car son interprétation ne dépend pas d'un contexte particulier, comme c'est le cas pour telly boys.

Nous avons vu plus haut que les adjectifs composés ne présentent pas le même effet de connivence que les noms composés, et mettons maintenant ceci en rapport avec le fait qu'ils comportent moins d'implicite. Ils sont moins synthétiques que les noms composés dans la mesure où ils semblent dire tout ce qu'il y a à dire (shell-shocked par exemple, équivaut à shocked with a shell). Ils sont donc accessibles à tous, et non pas seulement à certaines personnes qui auraient accès à l'information manquante.

En quoi consiste donc la condensation des adjectifs composés? Comme on l'a vu, le processus de formation des adjectifs composés ne semble pas impliquer la disparition d'éléments lexicaux. Seuls quelques mots grammaticaux disparaissent, par exemple les prépositions (spotlighted plutôt que lighted with a spot), ou les déterminants (hornlike plutôt que like $\underline{a}$ horn). Parfois même l'ordre syntaxique est respecté (sprawled out ou held out provenant respectivement des verbes spawl out et hold out, offscreen auquel on peut comparer off the screen), ce qui témoigne d'une réélaboration minimale. Le plus souvent cependant on observe une antéposition par rapport à la formule plus développée, comme c'est le cas pour trendy-looking ou austere-looking (ordre inversé par rapport à, respectivement, she looks trendy, she looks austere); ce remaniement reste toutefois minimal.

\section{Conclusion : vers une stylistique des parties du discours}

Les données présentées ici semblent indiquer que les noms composés sont brefs, tandis que les adjectifs composés le sont moins. Encore une fois, rappelons que nous ne comparons pas la longueur des adjectifs composés à celle des noms composés, mais la longueur des noms composés à celle d'un discours sous-jacent, et la longeur des adjectifs composés à celle d'une séquence syntaxique. Cette brièveté, parfois, se remarque, et est porteuse d'un effet de style. Certains noms composés en effet paraissent brefs simplement parce que la situation porte à attendre une séquence plus longue, notamment lorsque ces noms réfèrent à des réalités inexistantes ou surprenantes, qui mériteraient de ce fait plus ample présentation. Nous avons également vu que les noms composés qui «affichent» leur brièveté mettent en valeur le caractère partagé de la dénomination.

Dans la perspective d'une approche sémantique des parties du discours, l'étude des mots construits permet de mieux comprendre les catégories grammaticales (nominale et adjectivale en l'occurrence). En d'autres termes, si les noms composés sont plus brefs que les adjectifs composés, cela nous dit certainement quelque chose sur le nom en tant que partie du discours. Il nous reste maintenant à interpréter cette plus forte contrainte de la brièveté dans le domaine nominal. 
Nous supposons qu'elle est due au rôle catégorisant du nom [Wierzbicka 1988: 463497]. En effet, on ne discute généralement pas de l'appartenance d'un référent à une catégorie, mais plutôt des propriétés du référent. Si je dis par exemple This book is interesting on peut me répondre: No, it's not interesting (où l'on débat d'une propriété du livre), mais moins facilement?(No) it's not a book (où le débat porterait sur la dénomination). Le nom, en tant qu'il inscrit le référent dans une catégorie, fait l'objet d'un accord. Il est alors compréhensible que le nom soit caractérisé par la brièveté, qui apparaît comme garante du caractère communautaire de la dénomination. En effet, ce qu'on ne développe pas (ce qui est dit brièvement) est normalement ce qui va de soi et ce sur quoi on s'accorde. Au-delà du phénomène de composition, nous touchons aux différences entre l'adjectif et le nom en tant que parties du discours.

\section{Bibliographie}

BAUer L., English Word-Formation, Cambridge, Cambridge University Press, 1983.

Downing P.A., "On the Creation and Use of English Compound Nouns", Language 53, Waverly Press, 1977 : 810-842.

KRIPKE S., La logique des noms propres, traduit de l'anglais par P. Jacob et F. Recanati, Paris, Les Editions de Minuit, (1980) 1982.

LEVI J., The Syntax and Semantics of Complex Nominals, New-York, Academic Press, 1978.

MoLINIE G., Eléments de stylistique française, Paris, PUF, 1986.

Mignot E., Recherches sur les noms composés de type nom + nom en anglais contemporain, thèse de doctorat, Université Paris IV, sans éd., 2001.

WierzbickA A., The Semantics of Grammar, Amsterdam, Philadelphie, John Benjamins Publishing Company, 1988.

\section{Références des exemples cités}

Allen W., Four Films of Woody Allen, Londres, Boston, Faber and Faber, $1983 .{ }^{12}$

Bronte E., Wuthering Heights, Londres, Penguin Books, (1847) 2003.

Waterson B., The Authoritative Calvin and Hobbes, Kansas City, Andrews and McMeel, 1990.

Sillitoe A., The Loneliness of the Long Distance Runner, Londres, HarperCollins Publishers, 1959.

\footnotetext{
${ }^{12}$ Nous avons utilisé les scénarios de Annie Hall (pages 1 à 109) et Manhattan (pages 179 à 276).
} 